\title{
Cooperative control of perpendicular magnetic anisotropy via crystal structure and orientation in freestanding $\mathrm{SrRuO}_{3}$ membranes
}

Zengxing Lu $u^{1,2}$, Yongjie Yang ${ }^{1,2,3}$, Lijie Wen ${ }^{1,2,4}$, Jiatai Feng ${ }^{1,2}$, Bin Lao ${ }^{1,2}$, Xuan Zheng ${ }^{1,2,5}$, Sheng Li , $^{1,2}$, Kenan Zhao ${ }^{1,2}$, Bingshan Cao ${ }^{1,2,6}$, Zeliang Ren ${ }^{1,2,6}$, Dongsheng Song ${ }^{7}$, Haifeng Du $\mathbb{D}^{8}$, Yuanyuan Guo ${ }^{3}$, Zhicheng Zhong ${ }^{1,2,9}$, Xianfeng Hao $\mathbb{D}^{4 凶}$, Zhiming Wang ${ }^{1,2,9 凶}$ and Run-Wei $\mathrm{Li}^{1,2,9}$

Flexible magnetic materials with robust and controllable perpendicular magnetic anisotropy (PMA) are highly desirable for developing flexible high-performance spintronic devices. However, it is still challenge to fabricate PMA films on polymers directly. Here, we report a facile method for synthesizing single-crystal freestanding $\mathrm{SrRuO}_{3}$ membranes with controlled crystal structure and orientation using water-soluble $\mathrm{Ca}_{3-x} \mathrm{Sr}_{x} \mathrm{Al}_{2} \mathrm{O}_{6}$ sacrificial layers. Through cooperative effect of crystal structure and orientation, flexible membranes reveal highly tunable magnetic anisotropy from in-plane to out-of-plane with a remarkable PMA energy of $7 \times$ $10^{6} \mathrm{erg} \cdot \mathrm{cm}^{-3}$. First-principle calculations reveal that the underlying mechanism of PMA modulation is intimately correlated with structure-controlled Ru 4d-orbital occupation, as well as spin-orbital matrix element differences, dependent on the crystal orientation. In addition, even after 10,000 bending cycles, the PMA keeps stable, indicating a robust magnetism reliability in the prepared films. This work provides a feasible approach to prepare the flexible oxide films with strong and controllable PMA.

npj Flexible Electronics (2022)6:9; https://doi.org/10.1038/s41528-022-00141-3

\section{INTRODUCTION}

Flexible electronic/spintronic devices, with bendable and lightweight features, greatly expand the boundaries of spintronic/ electronic applications and are gradually changing personal habits in our daily life $\mathrm{e}^{1-6}$. As one of the core researching goals of the flexible spintronics/electronics, developing high-performance devices is highly coveted ${ }^{4-6}$. In this regard, integrating perpendicular magnetic anisotropy (PMA) to flexible devices shows tremendous potential. The PMA shows advantage in enhancing device density and lowering energy consumption while preserving superior thermal stability ${ }^{7-9}$. Moreover, controlling and tailoring the PMA on purpose plays a significant role in the formation of emergent spin textures such as chiral domain walls and magnetic skyrmions, which are considered as important ingredient for next-generation spintronic devices ${ }^{10,11}$. Currently, although a great number of the flexible PMA materials have been synthesized directly on flexible bases ${ }^{4,12-14}$, the limitations of the polymer substrates, such as low melting point and large roughness, hinder to obtain films with high-quality and robust PMA using the direct deposition technique ${ }^{15,16}$. Hence, it is desirable to develop facile method for the synthesis of high-quality flexible materials with a strong and controllable PMA.

$\mathrm{SrRuO}_{3}$ (SRO) is an attractive candidate for realizing flexible PMA-based spintronic materials and devices. It possesses itinerant ferromagnetism and strong spin-orbit coupling (SOC) simultaneously, resulting in an impressive magnetic crystalline anisotropy
(MCA) with an anisotropy energy (MAE) above $10^{6} \mathrm{erg} \mathrm{cm}^{-3}$ 17-20. Moreover, the SRO is a $4 d$ transition metal oxide (TMO), which has complex interactions between multiple degrees of freedom, and can trigger multiple types of phase transformations controlled by various external stimuli ${ }^{21-24}$. These features offer an opportunity to realize the strong and controllable PMA in the freestanding SRO membranes. Nowadays, for synthesizing the flexible films, one of the promising methods is to utilize sacrificial buffer layers through a combination of lift-off and thin-film transfer techniques 25,26 . Pioneered by Lu et al. in $2016^{27}$, the water-soluble $\mathrm{Sr}_{3} \mathrm{Al}_{2} \mathrm{O}_{6}$ (SAO) sacrificial layer has been widely used to synthesize a variety of freestanding crystalline TMOs membranes with millimeter-scale lateral size, controllable thickness down to ultrathin limit, and extremely large tolerable strain ${ }^{28-36}$. Moreover, different crystalline-oriented oxide membranes can be readily fabricated by changing the substrate crystal orientation, which provides a feasible way to control the properties of oxide membranes $24,35,37,38$. In addition, by controlling the thickness and composition of the sacrificial layer, it can further modulate the properties of freestanding TMOs membranes ${ }^{39,40}$.

In this work, using the $\mathrm{SAO}$ and $\mathrm{Ca}_{1.5} \mathrm{Sr}_{1.5} \mathrm{Al}_{2} \mathrm{O}_{6}$ (CSAO) as sacrificial layers, we prepare freestanding SRO films with different growth orientations, and realize a strong and highly tunned PMA. Intriguingly, the crystal structures of flexible membranes are tetragonal and orthorhombic when deposited with the SAO and CSAO, respectively. The different structures have a great influence

\footnotetext{
${ }^{1}$ CAS Key Laboratory of Magnetic Materials and Devices, Ningbo Institute of Materials Technology and Engineering, Chinese Academy of Sciences, Ningbo 315201, China.

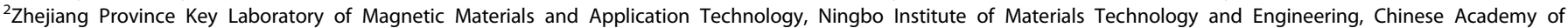
Sciences, Ningbo 315201, China. ${ }^{3}$ School of Materials and Metallurgy, University of Science and Technology Liaoning, Anshan 114051 , China. ${ }^{4}$ Key Laboratory of Applied Chemistry, College of Environmental and Chemical Engineering, Yanshan University, Qinhuangdao 066004, China. ${ }^{5}$ University of Nottingham, Ningbo 315201 , China. ${ }^{6}$ Nano Science and Technology Institute, University of Science and Technology of China, Hefei 230026, China. ${ }^{7}$ Institutes of Physical Science and Information Technology, Anhui

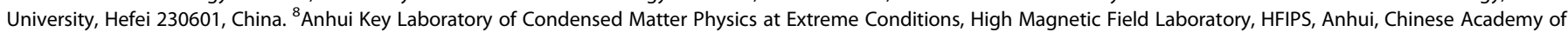
Sciences, Hefei 230031, China. ${ }^{9}$ Center of Materials Science and Optoelectronics Engineering, University of Chinese Academy of Sciences, Beijing 100049, China.

凶email: xfhao@ysu.edu.cn; zhiming.wang@nimte.ac.cn
} 


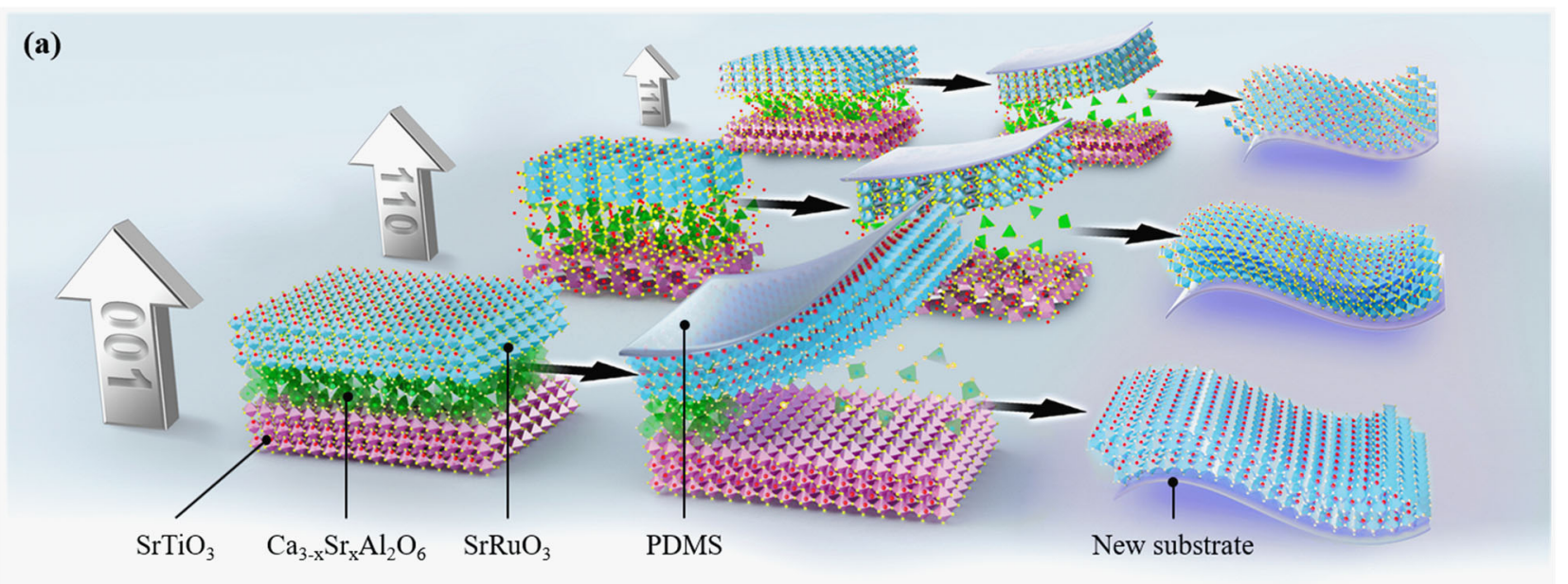

As-grown

Released

Transferred

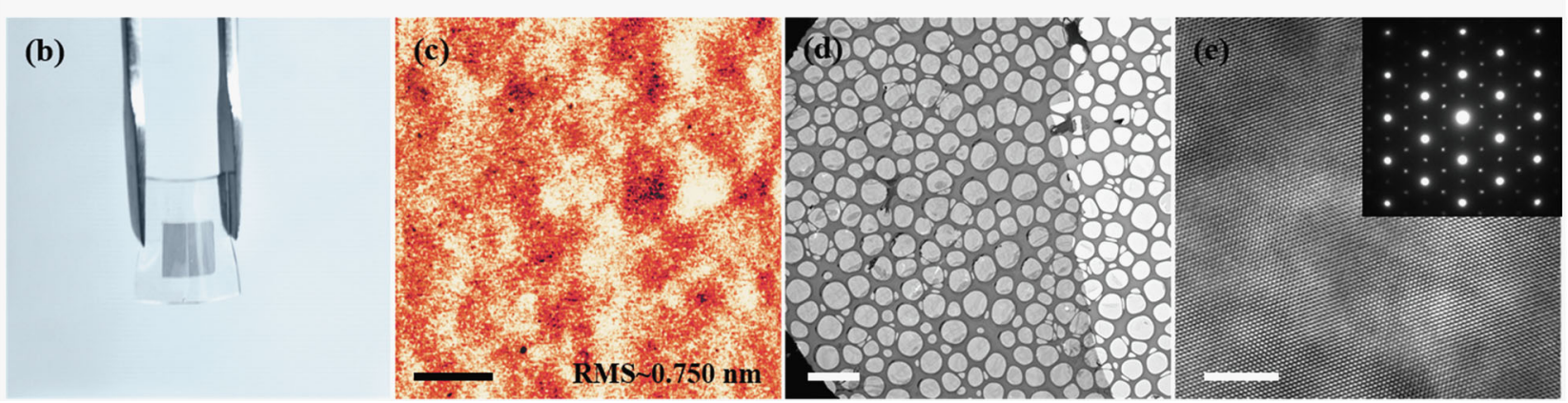

Fig. 1 Preparation of flexible SRO membranes with different crystal orientations. a Schematic illustrating the heterostructure growth, liftoff and transfer process for SRO membranes with different orientations. During the preparation, the water-soluble $\mathrm{Sr}_{3} \mathrm{Al}_{2} \mathrm{O}_{6}$ is used as sacrificial layers. b Flexible SRO membranes (gray area) transferred on soft PDMS. c An AFM image of the freestanding SRO membrane with a sub-nanometer roughness (RMS). Scale bar is $1 \mu \mathrm{m}$. d Low-magnification and e atomically resolved plane-viewed HR-TEM image of [111]oriented freestanding SRO membrane transferred on a TEM grid with holey carbon film. Scale bars are $5 \mu \mathrm{m}$ and $5 \mathrm{~nm}$ in (d) and (e), respectively. The inset in (e) shows the SAED pattern.

on the PMA and the effect becomes more prominent with the collaboration of the orientation. By altering the growth orientation of the films, first, the easy-axis of the tetragonal SRO is controlled. The [110]- and [111]-oriented films show in-plane MA (IMA) while the [001]-film owns a PMA. In contrary, for the orthorhombic SRO, the PMA is the protagonist in all of the three oriented films. Secondly, the PMA can be enlarged closely to $10^{7} \mathrm{erg} \cdot \mathrm{cm}^{-3}(7 \times$ $10^{6} \mathrm{erg} \cdot \mathrm{cm}^{-3}$ ) in the [110]-oriented orthorhombic SRO. According the first-principle calculations, we find that the $\mathrm{d}$-orbit band and MA of the Ru atom in the SRO are tightly affected by the orientation and crystal structure, leading the orientation- and structure-dependent MA. Finally, our films also exhibit reliable magnetism against the bending test. These results demonstrate that the sacrificial layer is a powerful approach to fabricate highquality flexible oxide films, and the structure and orientation are of importance to realize the strong and controllable PMA in the prepare membranes.

\section{RESULTS AND DISCUSSION Sample synthesis}

Figure 1a shows the schematic process for fabricating flexible SRO films with different orientations, i.e., [001], [110], and [111]. SRO/ SAO bilayers are deposited on the [001]-, [110]-, and [111]oriented $\mathrm{SrTiO}_{3}$ (STO) substrates using pulsed laser deposition (PLD) (seen details in the METHODS). During the deposition, the thickness of the film is monitored by the reflection high-energy electron diffraction (RHEED) and controlled with 30 unit cells (u.c.) and 40 u.c. for the SAO and SRO, respectively. Then, the bilayers are immersed in pure water to etch the sacrificial SAO layers and separate the SRO membranes. During the etching process, flexible PDMS is used as a support layer to mechanically stabilize the films ${ }^{27}$. After releasing, the SRO/PDMS structures are transferred on desired rigid or flexible substrates. Then the freestanding membranes remain on the fresh substrates after peeling off the PDMS via thermally releasing. Finally, we obtain millimeter-scale and complete flexible SRO films as shown in Fig. 1b. The surface and crystallization quality of the freestanding membranes are characterized by RHEED, atomic force microscopy (AFM), highresolution transmission electron microscopy (HR-TEM) and selected-area electron diffraction (SAED) as shown in Fig. 1c-e and Supplementary Figure 1. These measurements show the SRO membranes are smooth with sub-nanometer roughness and high crystallization quality.

\section{Structural analyses}

To explore the sacrificial layer influence on the magnetic properties of freestanding SRO membranes, we utilize different $\mathrm{Ca}_{3-\mathrm{x}} \mathrm{Sr}_{\mathrm{x}} \mathrm{Al}_{2} \mathrm{O}_{6}$ as sacrificial layers. In Fig. 2a, we compare lattice parameters of various $\mathrm{Ca}_{3-\mathrm{x}} \mathrm{Sr}_{x} \mathrm{Al}_{2} \mathrm{O}_{6}$ sacrificial layers with bulk SRO and STO. We choose $\mathrm{Ca}_{1.5} \mathrm{Sr}_{1.5} \mathrm{Al}_{2} \mathrm{O}_{6}(\mathrm{x}=1.5, \mathrm{CSAO})$ with a lattice constant $a_{\mathrm{CSAO}}=4 \times 3.87 \AA$, smaller than SRO and STO, while SAO has a larger lattice constant $\left(a_{\mathrm{SAO}}=4 \times 3.96 \AA\right)$ than SRO and $\mathrm{STO}^{27,37,40}$. These different sacrificial layers can impose dissimilar strain in the SRO films. Here, we denote the SRO grown with SAO and CSAO as $\mathrm{SRO}_{\mathrm{SAO}}$ and $\mathrm{SRO}_{\mathrm{CSAO}}$, respectively. Supplementary Figure 2 and Supplementary Figure 3 show smooth surface and 
(a)

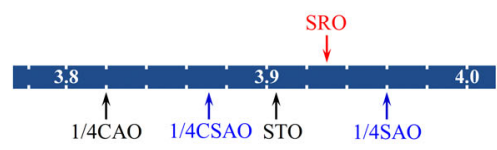

Orthorhombic SRO Tetragonal SRO
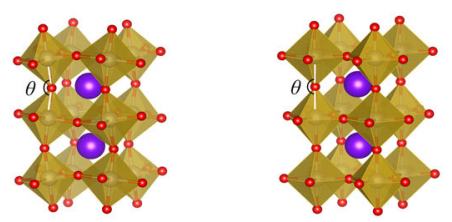

[001] S-SRO

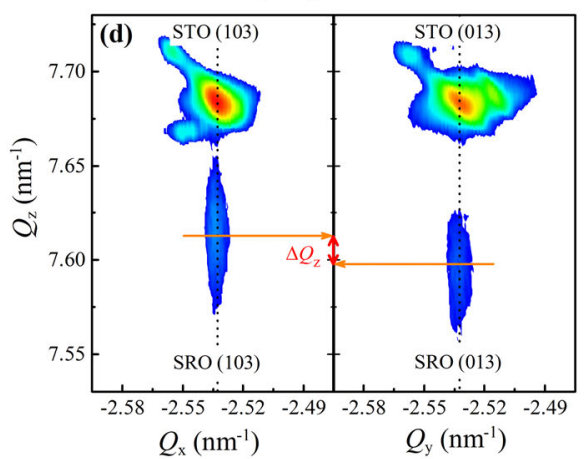

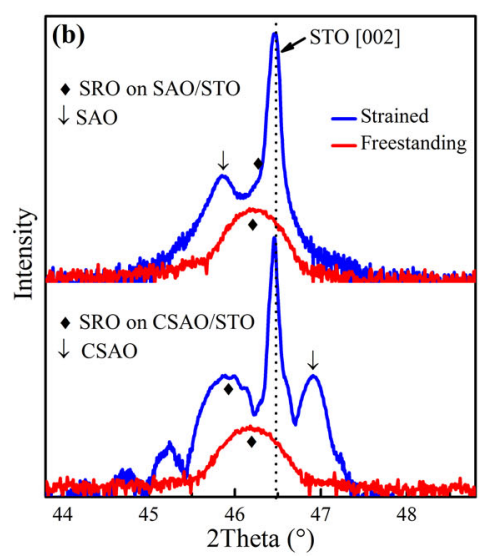

[001] F-SROsao

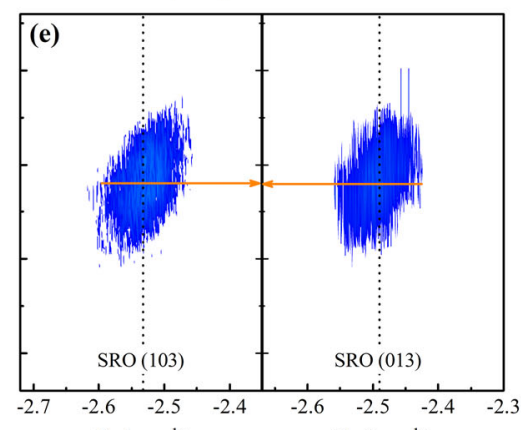

$Q_{\mathrm{x}}\left(\mathrm{nm}^{-1}\right)$

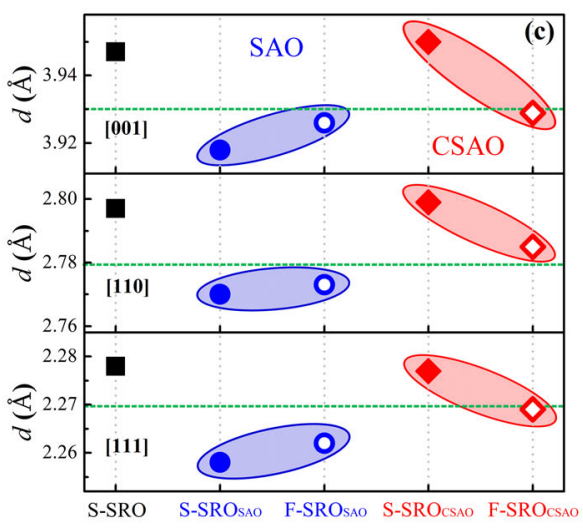

[001] F-SROCSAO

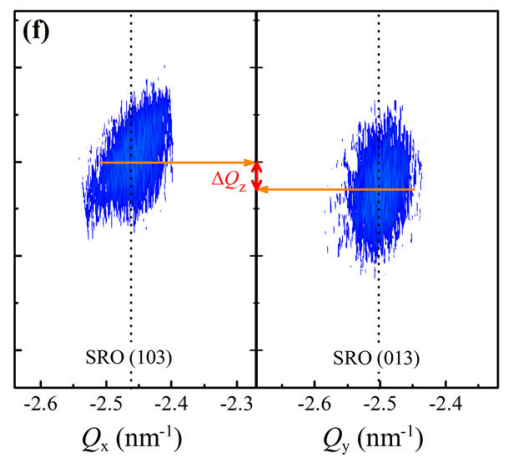

Fig. 2 Crystalline structure of epitaxial freestanding SRO films grown with different sacrificial layers. a Comparative lattice constants $(\AA)$ of the pseudocubic SRO, sacrificial layers (i.e., SAO and CSAO) and STO substrate. b XRD $2 \theta-\omega$ scans of the strained and freestanding SRO films with the SAO (top) and CSAO (bottom). c Interplanar distance $d$ of the strained and freestanding [001]-, [110]- and [111]-oriented SRO films grown on STO, SAO/STO (blue), and CSAO/STO (red). d-f Reciprocal space maps around the (103) and (013) peaks for the (d) [001]-oriented SRO films grown on STO substrate, [001]-oriented freestanding SRO films grown with (e) SAO and (f) CSAO. S-SRO, S-SRO SAO, and S-SRO $_{\text {CSAO }}$ represent the strained SRO without sacrificial layer, with SAO and CSAO buffer layers. F-SRO SAO $_{\text {and F-SRO }}$ CSAO represent the freestanding SRO grown with SAO and CSAO.

high crystalline quality of the $\mathrm{SRO}_{\mathrm{CSAO}}$, respectively, indicating the highquality of the freestanding $\mathrm{SRO}_{\mathrm{CSAO}}\left(\mathrm{F}-\mathrm{SRO}_{\mathrm{CSAO}}\right)$ as observed in the freestanding $\mathrm{SRO}_{\mathrm{SAO}}\left(\mathrm{F}-\mathrm{SRO}_{\mathrm{SAO}}\right)$. The X-ray diffraction (XRD) scans in Fig. $2 \mathrm{~b}$ and Supplementary Figure 5 only show the peaks of the SRO, SAO/CSAO, and STO substrate, revealing epitaxial growth and single phase of the as-grown (strained) and released (freestanding) SRO films.

More importantly, the systematical XRD measurements confirm the stress strain related to the sacrificial layers. According to the $2 \theta-\omega$ scan curves, we calculate the interplanar distance $(d)$ using the Bragg formula. As shown in Fig. 2c, for the [001]-case, the $d$ of the strained $\mathrm{SRO}_{\mathrm{SAO}}\left(\mathrm{S}-\mathrm{SRO}_{\mathrm{SAO}}\right)$ is $3.918 \AA$ which is smaller than that of bulk pseudocubic SRO ${ }^{18,41}(d=3.93 \AA$, marked with a green line), indicating that the SRO undergoes a tensile strain induced by the large lattice constant of the SAO. In contrast, the strained $\mathrm{SRO}_{\mathrm{CSAO}}\left(\mathrm{S}-\mathrm{SRO}_{\mathrm{CSAO}}\right.$ ) grown with CSAO is compressed and its $d$ is elongated out-of-plane to $3.95 \AA$. These observations are also observed in the [110]- and [111]-oriented SRO films as shown in Fig. 2c and Supplementary Figure 4, demonstrating that the SRO films are tensely and compressively strained when grown with the SAO and CSAO sacrificial layer, respectively. Next, we carry out the XRD scans of the freestanding SRO (F-SRO) films attached on the PDMS. Figure $2 \mathrm{~b}$ demonstrates that the peaks of SRO membranes shift to left (right) after dissolving the SAO (CSAO) sacrificial layers, further confirming the tensile (compressive) strain in the as-grown $\mathrm{SRO}_{\mathrm{SAO}}\left(\mathrm{SRO}_{\mathrm{CSAO}}\right)$ films, respectively. The interplanar distances $d$ of the $\mathrm{SRO}_{\mathrm{SAO}}$ and $\mathrm{SRO}_{\text {CSAO }}$ films increase and decrease to $3.926 \AA$ and $3.929 \AA$ after lift-off, respectively. Correspondingly, the similar changes of the $d$ are also observed in [110]- and [111]-oriented membranes, as summarized in Fig. 2c. Obviously, there is a stress release in the freestanding SRO films. However, it should be noted that the $\mathrm{F}_{-} \mathrm{SRO}_{\text {SAO }}$ membranes have smaller $d$ compared to the $\mathrm{F}_{-\mathrm{SRO}} \mathrm{CSAO}_{\mathrm{O}}$ membranes regardless of the crystal orientation. Such a difference may suggest a structural transition in SRO films induced by different sacrificial layers.

To verify the structural transition, we perform $\mathrm{X}$-ray reciprocal space mapping measurements. As shown in Fig. 2d, the mapping around (103)- and (013)-diffraction peaks of the SRO grown on STO without sacrificial layers reveal that (103)-peak of the SRO possess higher a $Q_{z}$ value than the (013)-peak $\left(\Delta Q_{z} \neq 0\right)$. This indicates that the SRO is orthorhombic with a monoclinic distortion, in consistent with previous literatures ${ }^{18,42-45}$. Similar results are observed for the strained and freestanding SRO films grown with the CSAO, in which different $Q_{z}$ values for the (103)and (013)-diffraction peaks are observed (Supplementary Fig. 6b and Fig. 2f). This implies S-SRO $\mathrm{CSAO}_{\text {a }}$ and $\mathrm{F}-\mathrm{SRO}_{\mathrm{CSAO}}$ possess orthorhombic structure too. However, by comparing the peaks position for the films grown with the SAO (Supplementary Fig. 6a and Fig. 2e), it is found that the (103)- and (013)-peaks have same $Q_{z}$ values $\left(\Delta Q_{z}=0\right)$, indicating that the S-SRO SAO and F-SRO SAO are tetragonal ${ }^{18,42-45}$. These results demonstrate that the structural transition can be engineered via altering the sacrificial layers, as shown in Fig. 2a. It is consistent with the literatures, in which the SRO films can form orthorhombic or tetragonal phases under the stress imposed by the substrate ${ }^{18,45}$, as we observed in the SAO/ STO and CSAO/STO heterostructures. Both phases are almost degenerate in energy ${ }^{46,47}$. On the other hand, these two crystal 

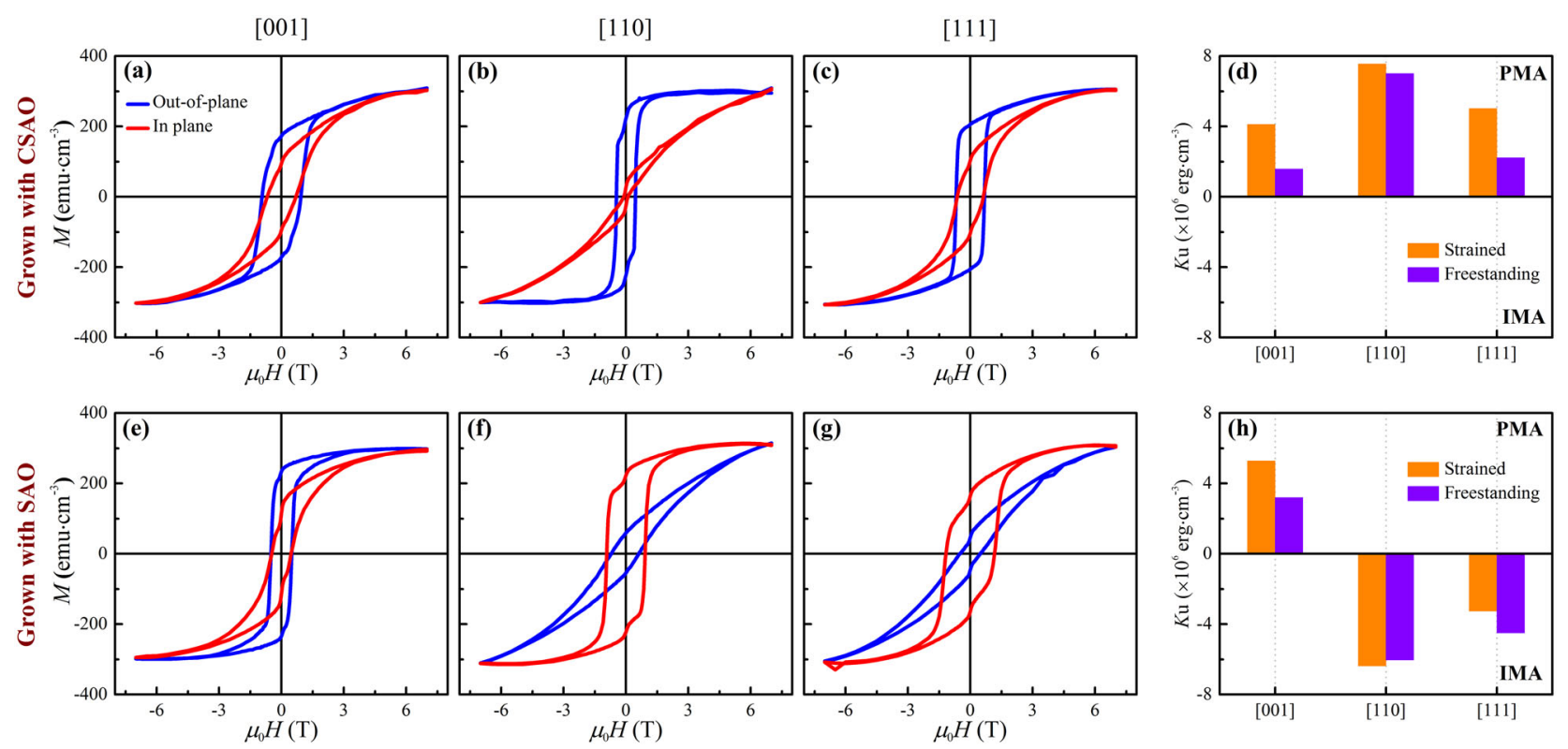

Fig. 3 MA of the freestanding SRO films modulated by the crystal orientation and structure. a-c and e-g MA of the (a) and (e) [001]-, (b) and (f) [110]-, (c) and (g) [111]-oriented freestanding SRO films grown with the CSAO and SAO, respectively. d, $\mathbf{h}$ MAE dependent on the orientation in the freestanding SRO films grown with the (d) CSAO and (h) SAO, respectively. PMA and IMA in (d) and (h) are the abbreviations of perpendicular and in-plane magnetic anisotropy, respectively.

structures are belonging to different space groups that prohibit direct transformation among each other ${ }^{47}$. Therefore, these different crystal structures can be stabilized by the strain imposed by the sacrificial layers, and the structural phases could be kept even being peeled off from the substrates, leading the structural transition observed above.

\section{Magnetic anisotropy}

The crystal orientations and observed structural transition have a remarkable influence toward the magnetic properties of flexible SRO films. To explore the impacts, magnetic hysteresis $\left(M-\mu_{0} H\right)$ measurements were performed with the magnetic field in and out of the sample plane. As shown in Fig. 3, the magnetization is more easily reversed and saturated in [110] $\mathrm{F}_{-} \mathrm{SRO}_{\mathrm{CSAO}}$ membranes (Fig. 3a-c) among the three orthorhombic samples, while it is easier in [001] tetragonal F-SRO ${ }_{\mathrm{SAO}}$ membranes (Fig. 3e-f). Moreover, the SRO membranes exhibit large MAEs above $1 \times$ $10^{6} \mathrm{erg} \cdot \mathrm{cm}^{-3}$ (Fig. $3 \mathrm{~d}, \mathrm{~h}$ ), confirming a robust MCA in SRO due to its strong $\mathrm{SOC}^{48}$. Intriguingly, the impacts of crystal orientations and structure on the MA are more pronounced. As shown in Fig. $3 a-c$, the orthorhombic F-SRO ${ }_{C S A O}$ membrane, obtained with the CSAO sacrificial layer, shows an orientation-dependent PMA. The [110]-oriented $\mathrm{F}-\mathrm{SRO}_{\mathrm{CSAO}}$ membrane owns the largest anisotropy energy $\left(\mathrm{MAE}, K_{\mathrm{u}}\right)$ of $7 \times 10^{6} \mathrm{erg} \cdot \mathrm{cm}^{-3}$, which is 4.46 and 3.15 times of the films grown along [001] $(1.57 \times$ $\left.10^{6} \mathrm{erg} \cdot \mathrm{cm}^{-3}\right)$ and [111] $\left(2.22 \times 10^{6} \mathrm{erg} \cdot \mathrm{cm}^{-3}\right)$ directions, as summarized in Fig. $3 \mathrm{~d}$. In contrast, while in the tetragonal F-SRO $\mathrm{SAO}_{\mathrm{SO}}$ membrane grown with the SAO, not only the MAE is orientationdependent, but also the magnetic easy-axis is controlled by the orientation (Fig. 3e-h). At first, the $\left|K_{\mathrm{u}}\right|$ of the [110]-film is the largest of $6.05 \times 10^{6} \mathrm{erg} \cdot \mathrm{cm}^{-3}$ which is 1.9 and 1.34 times of the [001]- and [111]-films. Moreover, by altering the orientation from [001] to [110] and [111], the easy-axis rotates from out-ofplane to in-plane direction. These results demonstrate that the MA of the freestanding SRO film is not only dependent on the crystal orientation, but also the crystal structure which can be controlled further by the sacrificial layers.

\section{First-principle calculations}

To understand the cooperative effects of crystal orientation and structure on the MA of freestanding SRO membranes, we perform first-principle density functional theory (DFT) calculations of the SRO MAE with the orthorhombic and tetragonal structure, as illustrated in Fig. 2a (see details in METHODS). The calculated MAE is defined as the energy difference between the magnetization along the out-of-plane and in-plane. The positive and negative values of MAE represent perpendicular and in-plane MA, respectively. According to Fig. $4 a, d$, firstly, the total energy calculation indicates that the SRO has a pronounced MCA with a high-energy magnitude around $\sim 1 \mathrm{meV} \cdot \mathrm{u} \cdot \mathrm{c}^{-1} \cdot\left(\sim 10^{7} \mathrm{erg} \cdot \mathrm{cm}^{-3}\right)$. Secondly, both of the [110] orthorhombic and tetragonal phases have the largest MAE among three different crystal orientations. More importantly, the magnetic easy-axis can been tuned by the crystal orientation and structure synergistically. For the orthorhombic SRO, it shows a MA with a vertical easy-axis, exhibiting PMA for all three orientations. While for the tetragonal structure, the easy-axis is flipped from the out-of-plane direction to the plane when changing the orientation from [001] to [110] and [111]. These results are well consistent with our experimental observations, demonstrating the cooperative effect of crystal orientation and structure on modulating not only the magnitude of magnetic anisotropy energy but also the direction of the magnetic easy-axis in SRO.

To gain further insight into the cooperative modulation effect, we have performed element- and orbital-projected MAE calculations for both orthorhombic and tetragonal SRO based on a second-order perturbation theory (see details in METHODS). The element-resolved calculations show large MAEs $\left(\sim 1 \mathrm{meV} \cdot\right.$ u.c. $\left.^{-1}\right)$ of the Ru atoms (Ru-MAEs) in both structural phases, as shown in Supplementary Figure 8. This indicates that SRO possesses a large single-ion anisotropy due to the strong spin-orbital interactions, in consistent with results of the $\mathrm{SrIrO}_{3} / \mathrm{La}_{0.67} \mathrm{Sr}_{0.33} \mathrm{MnO}_{3}$ heterostructures $^{49}$. Fig. 4b, e show the corresponding orbital-projected RuMAEs for the [110]-oriented orthorhombic and tetragonal SRO, respectively. In the orthorhombic phase, the $\left(d_{\mathrm{xz}}, d_{\mathrm{yz}}\right)$ matrix element makes an outstanding out-of-plane contribution to the Ru-MAE, leading a PMA (Fig. 4b) with a MAE of 0.937 meV.u.c. ${ }^{-1}$. 

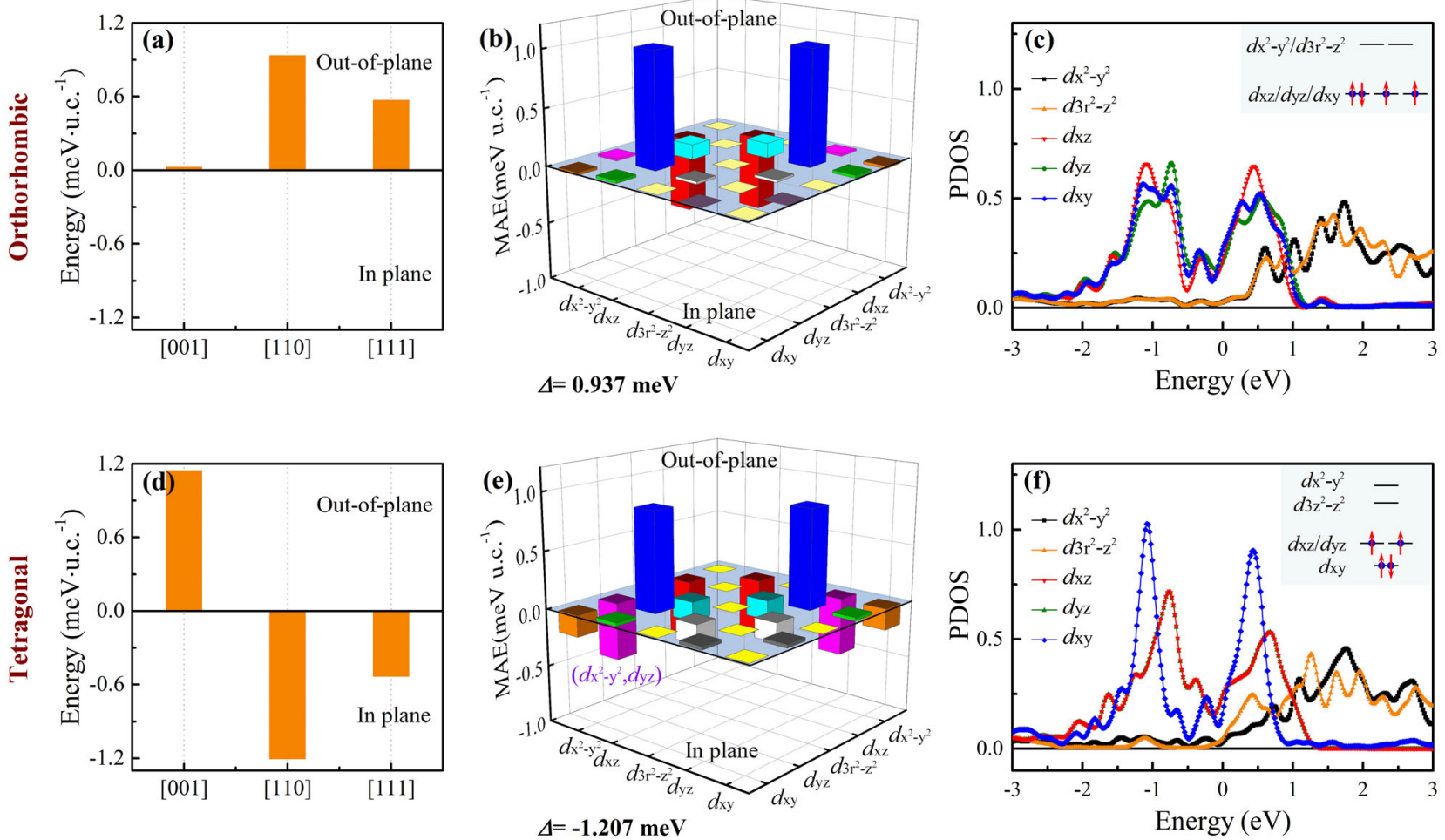

Fig. 4 Mechanism of the orientation- and structure-dependent MA. a d The MAE of the (a) orthorhombic and (d) tetragonal SRO dependent on the orientation, respectively. b, e The $d$ orbit-resolved MAE of the Ru atoms in [110]-oriented (b) orthorhombic and (e) tetragonal SRO. c, f The $d$ orbit-resolved PDOSs of the Ru atoms in (c) orthorhombic and (f) tetragonal SRO. The insets in (c) and (f) shows the $d$-orbit energy band structures modulated by the structure.

However, for the tetragonal SRO, although the $\left(d_{\mathrm{xz}}, d_{\mathrm{yz}}\right)$ keeps positive, most of the elements become negatively inducing an IMA with a MAE of -1.207 meV.u.c. ${ }^{-1}$ (Fig. 4e). It confirms that the tunable MA of SRO membranes are indeed controlled by Ru-MAEs through crystal orientation and structure. First-principle DFT calculations show the partial density of states (PDOS) around the Fermi level are solely derived from $\mathrm{Ru}$ atoms as shown in Fig. $4 \mathrm{c}$, $\mathrm{f}$. In SRO, there is four electrons occupied the $t_{2 \mathrm{~g}}$ orbitals due to the crystal field splitting. The symmetrical reduction gives rise to the rotation and tilting of the $\mathrm{RuO}_{6}$ octahedron in orthorhombic SRO, alters the individual Ru-O-Ru bond length and angle, and then modifies the states of splitting and the electronic occupation, compared to the tetragonal phase. This structural modulation leads to a more uniform occupation of the $t_{2 g}$ orbital in the orthorhombic phase rather than in the tetragonal one. As shown in the inset of Fig. 4c, in the orthorhombic structure, the $t_{2 g}$ orbitals are almost three-fold degenerate. The degeneracy is partially lifted, however, in the tetragonal SRO. Two electrons are preferred occupied the $d_{x y}$ orbitals first, and the other electrons are located on the degenerate $d_{\mathrm{xz}} / d_{\mathrm{yz}}$ orbitals as illuminated in the inset of the Fig. $4 \mathrm{f}$. These structure-dependent orbital splitting directly influence the PDOS at the Fermi level, which in turn affect the Ru-MAEs.

\section{Bending tests}

For actual application, the flexibility of the freestanding films should satisfy the reliability demands of flexible devices ${ }^{50}$. To probe the reliability, a bending cycle test is taken on the [110]-oriented orthorhombic SRO sandwiched between two 50- $\mu \mathrm{m}$ PDMS films and adhered on a PI tape, forming a structure of PDMS/SRO/PDMS/PI. For the test, as shown in Fig. 5a, the testing structure is fixed by two molds which compresses the film with a radius of $2 \mathrm{~mm}$, and then flattens without tensile stress as one bending cycle. Next, the magnetization-dependent field and temperature $\left(M-\mu_{0} H\right.$ and $\left.M-T\right)$ are characterized as a criterion for the endurance. Figure $5 \mathrm{~b}$ shows the out-of-plane and in-plane $M-\mu_{0} H$ curves after various bending cycles. During the testing, there is negligible change in the $K_{\mathrm{u}}$, saturated and remanent magnetizations $\left(M_{S}\right.$ and $\left.M_{R}\right)$ as well as the Curie temperature $\left(T_{\mathrm{C}}\right)$, indicating that the flexible membranes possess stable magnetic properties even after 10,000 bending cycles, showing great potential for flexible devices.

In summary, we have successfully fabricated high-quality flexible SRO membranes with a strong and tunable PMA using the method of sacrificial layers. It is observed that the PMA, including MAE and magnetic easy-axis of the films, can be manipulated cooperatively by changing the growth orientation and sacrificial buffers, i.e., CSAO and SAO. The reason is that the crystal structure of the SRO film can be controlled by the buffers, and with the collaboration of the orientation. As a result, by optimizing the orientation along [110], we achieve a flexible PMA film with a maximum MAE of $7 \times$ $10^{6} \mathrm{erg} \cdot \mathrm{cm}^{-3}$ in orthorhombic SRO when grown with the CSAO. The first-principle calculations indicate that the $d$-orbit band and MA of the Ru atom in the SRO is tightly affected by the orientation and crystal structure, leading the orientation- and structuredependent MA. In addition, by performing a bending cycle test, the flexible membranes also show a good magnetism reliability.

Our work exemplifies that the water-soluble sacrificial layer is a powerful approach to prepare high-quality flexible electric/ magnetic films with reliable physics properties. Although, only millimeter-size samples have been fabricated currently ${ }^{29-31}$, this technique has shown a potential capability in large area and mass production in future, which is important to practical application. Additionally, the collaboration of the structure and orientation are efficiency to modulate the physics properties of functional materials, especially the PMA. The above strategies can be extended to a wide class of the oxide systems, and used to fabricate flexible oxide-based devices in future, especially to develop cryogenic electronics/spintronic, which is gaining great 
(a) One bending cycle
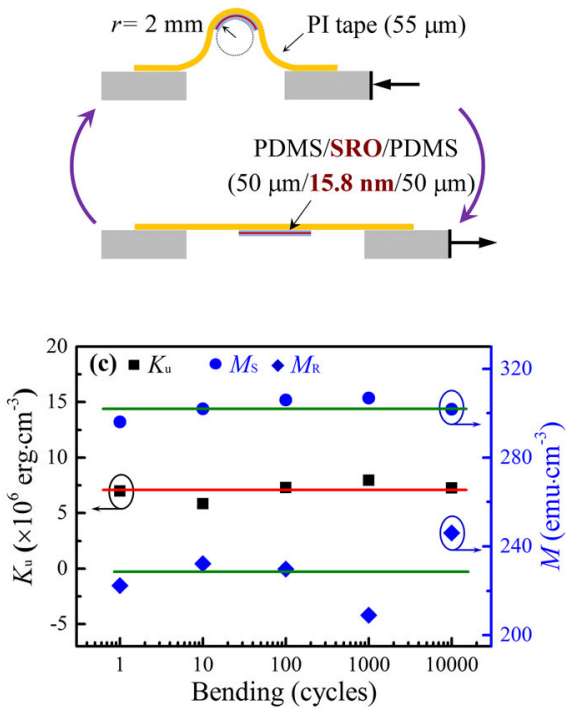
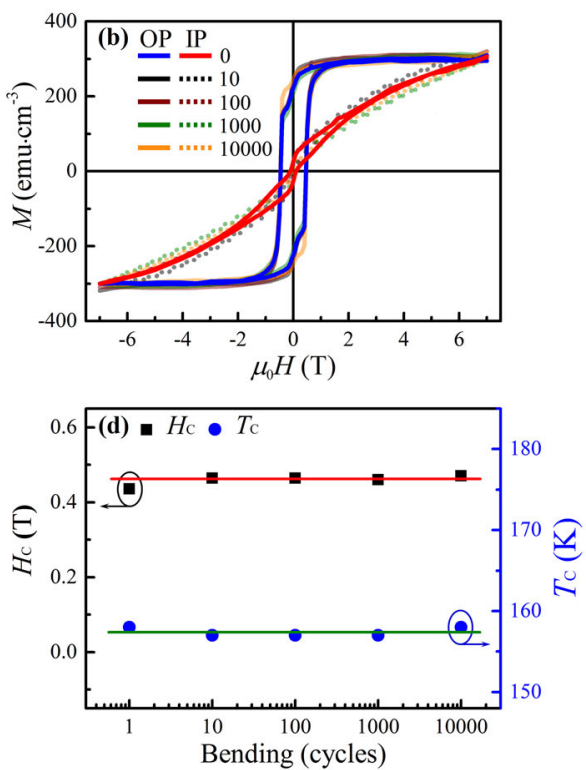

Fig. 5 The reliability of the flexible SRO films. a Sketch of the bending cycle test. $\mathbf{b}$ The $M-\mu_{0} H$ loops of films in flat state measured after different bending cycles. OP and IP are abbreviations of out-of-plane and in plane. $\mathbf{c}$ The plots of $K_{\mathrm{u}}$, saturated and remanent magnetizations $\left(M_{\mathrm{S}}\right.$ and $\left.M_{\mathrm{R}}\right)$ versus bending cycles. d The plots of out-of-plane coercive field and Curie temperature $\left(H_{\mathrm{C}}\right.$ and $\left.T_{\mathrm{C}}\right)$ versus bending cycles. During the test, the [110]-oriented flexible orthorhombic SRO film, sandwiched between two 50- $\mu \mathrm{m}$ PDMS films and adhered on a 55- $\mu \mathrm{m}$ PI tape, is used and bent to $2 \mathrm{~mm}$ radius.

anticipation to traverse the power/memory wall of classical room temperature computing ${ }^{51,52}$.

\section{METHODS}

\section{Film preparation}

The SAO/SRO and CSAO/SRO bilayers are deposited on different oriented STO substrates by pulsed laser deposition (PLD) using a $\operatorname{KrF}(\lambda=248 \mathrm{~nm})$ excimer laser. The SAO layer with a thickness of 30 u.c. is deposited on the [001]-STO substrate at a substrate temperature $T_{\text {sub }}=700^{\circ} \mathrm{C}$ with an oxygen pressure $P_{\mathrm{O}_{2}}=2 \times 10^{-3} \mathrm{mbar}$. The laser fluence is $2.0 \mathrm{~J} \cdot \mathrm{cm}^{-2}$, and the repetition rate is $4 \mathrm{~Hz}$. Subsequently, 40 u.c. SRO film is grown with the same laser flux at $T_{\text {sub }}=$ $700{ }^{\circ} \mathrm{C}$ and $\mathrm{PO}_{2}=0.1 \mathrm{mbar}$. With same pulse counts and preparation condition of the SAO and SRO, the [110]- and [111]-bilayers are prepared. With same methods, the oriented CSAO/SRO bilayers are fabricated. Here, the CSAO layers are deposited with same condition as the SAO.

\section{Film transfer}

The films are adhered on clean polydimethylsiloxane (PDMS), then immersed in pure water at room temperature for $12 \mathrm{~h}$ to dissolve the SAO and CSAO buffers completely. Then, the SRO/PDMS structures are lifted out and washed clean with acetone. Finally, we obtain the different oriented flexible SRO films adhered on the PDMS. It should be noted that the SRO films can be transferred on other substrates or supports, such as the $\mathrm{Si}, \mathrm{STO}$, or TEM grids after being baked at $90^{\circ} \mathrm{C}$ for $10 \mathrm{~min}$.

\section{Characterizations}

The information on the surface morphology, epitaxy quality, and crystal structure are examined by atomic force microscopy (AFM, Dimension 3100, Bruker) and X-ray diffraction (XRD) scan (D8 Discover, Bruker). The highresolution transmission electron microscopy and selected-area electron diffraction (HE-TEM and SAED, Talos F200CX, Thermal Fischer Scientific) are used to further characterize the crystalline quality. To perform the HE-TEM and SAED measurements for the flexible films, the freestanding SRO films are transferred on the TEM grids with holey carbon film. The magnetic properties are measured using superconducting quantum interference device (SQUIDVSM, Quantum Design) at $10 \mathrm{~K}$. To measure the magnetism of the flexible SRO, the films are transferred on the clean STO substrates. During bending cycle testing, the [110]-oriented orthorhombic SRO is sandwiched between two $50-\mu \mathrm{m}$ PDMS films then supported by a $55-\mu \mathrm{m}$ PI tape, constructing a structure of PDMS/SRO/PDMS/PI. Then, the testing structure is fixed by two molds which compresses the film with a radius of $2 \mathrm{~mm}$, and then flattens without tensile stress as one bending cycle. Next, the magnetizationdependent field and temperature $\left(M-\mu_{0} H\right.$ and $\left.M-T\right)$ are characterized as a criterion for the endurance.

\section{First-principle calculations}

First-principles calculations were performed within the Vienna ab initio simulation package (VASP) code ${ }^{53-55}$. In all the calculations, the exchangecorrelation potential was treated with the generalized gradient approximation (GGA) with the Perdew-Burke-Ernzerhof (PBE) functional ${ }^{56}$. The strong correlation effects for the Ru $4 d$ is taken into account by means of the GGA $+U$ approach and the on-site effective Coulomb interaction parameter $U_{\text {eff }}$ is taken as $2.0 \mathrm{eV}^{57}$. The cutoff energy was $500 \mathrm{eV}$ and $8 \times 8 \times 6 \mathrm{k}$-mesh was used in all simulations. To simulate the epitaxy, the horizontal lattice constants were fixed to those of the substrate, while the vertical one has been optimized until the force on each atom is smaller than $0.01 \mathrm{eV} \cdot \AA^{-1}$. Furthermore, the magnetic anisotropy energy is calculated by using the force theorem?

\section{DATA AVAILABILITY}

The data that support the findings of this study are available from the authors on reasonable request, see author contributions for specific data sets.

Received: 15 September 2021; Accepted: 6 January 2022; Published online: 08 February 2022

\section{REFERENCES}

1. Harris, K. D., Elias, A. L. \& Chung, H. J. Flexible electronics under strain: a review of mechanical characterization and durability enhancement strategies. J. Mater. Sci. 51, 2771-2805 (2015).

2. Liu, Y., He, K., Chen, G., Leow, W. R. \& Chen, X. Nature-inspired structural materials for flexible electronic devices. Chem. Rev. 117, 12893-12941 (2017).

3. Chen, X. \& Mi, W. Mechanically tunable magnetic and electronic transport properties of flexible magnetic films and their heterostructures for spintronics. J. Mater. Chem. C. 9, 9400-9430 (2021).

4. Makushko, P. et al. Flexible magnetoreceptor with tunable intrinsic logic for onskin touchless human-machine interfaces. Adv. Funct. Mater. 31, 2101089 (2021).

5. Karnaushenko, D. et al. High-performance magnetic sensorics for printable and flexible electronics. Adv. Mater. 27, 880-885 (2015). 
6. Loong, L. M. et al. Flexible MgO barrier magnetic tunnel junctions. Adv. Mater. 28, 4983-4990 (2016).

7. Dieny, B. \& Chshiev, M. Perpendicular magnetic anisotropy at transition metal/ oxide interfaces and applications. Rev. Mod. Phys. 89, 025008 (2017).

8. Ikeda, S. et al. A perpendicular-anisotropy CoFeB-MgO magnetic tunnel junction. Nat. Mater. 9, 721-724 (2010).

9. Mangin, S. et al. Current-induced magnetization reversal in nanopillars with perpendicular anisotropy. Nat. Mater. 5, 210-215 (2006).

10. Soumyanarayanan, A., Reyren, N., Fert, A. \& Panagopoulos, C. Emergent phenomena induced by spin-orbit coupling at surfaces and interfaces. Nature 539, 509-517 (2016).

11. Nagaosa, N. \& Tokura, Y. Topological properties and dynamics of magnetic skyrmions. Nat. Nanotechnol. 8, 899-911 (2013).

12. Vemulkar, T., Mansell, R., Fernández-Pacheco, A. \& Cowburn, R. P. Toward flexible spintronics: perpendicularly magnetized synthetic antiferromagnetic thin films and nanowires on polyimide Substrates. Adv. Funct. Mater. 26, 4704-4711 (2016).

13. Matsumoto, H., Ota, S., Koyama, T. \& Chiba, D. Control of magnetic anisotropy in a Co thin film on a flexible substrate by applying biaxial tensile strain. Appl. Phys. Lett. 118, 022406 (2021).

14. Ota, S. et al. Strain-induced reversible modulation of the magnetic anisotropy in perpendicularly magnetized metals deposited on a flexible substrate. Appl. Phys. Express 9, 043004 (2016).

15. Hassan, M. et al. Perpendicularly magnetized Co/Pd-based magneto-resistive heterostructures on flexible substrates. Nanoscale Adv. 3, 3076-3084 (2021).

16. Huang, J., Wang, H., Sun, X., Zhang, X. \& Wang, H. Multifunctional $\mathrm{La}_{0.67} \mathrm{Sr}_{0.33} \mathrm{MnO}_{3}$ (LSMO) thin films integrated on mica substrates toward flexible spintronics and electronics. ACS Appl. Mater. Interfaces 10, 42698-42705 (2018).

17. Qin, Q. et al. Emergence of topological hall effect in a $\mathrm{SrRuO}_{3}$ single layer. $A d v$. Mater. 31, 1807008 (2019).

18. Lu, W. et al. Strain engineering of octahedral rotations and physical properties of $\mathrm{SrRuO}_{3}$ films. Sci. Rep. 5, 10245 (2015)

19. Kim, B. \& Min, B. I. Termination-dependent electronic and magnetic properties of ultrathin $\mathrm{SrRuO}_{3}$ (111) films on $\mathrm{SrTiO}_{3}$. Phys. Rev. B 89, 195411 (2014).

20. Liu, L. et al. Current-induced magnetization switching in all-oxide heterostructures. Nat. Nanotechnol. 14, 939-944 (2019).

21. Hwang, H. Y. et al. Emergent phenomena at oxide interfaces. Nat. Mater. 11, 103-113 (2012)

22. Zubko, P., Gariglio, S., Gabay, M., Ghosez, P. \& Triscone, J.-M. Interface physics in complex oxide heterostructures. Annu. Rev. Condens. Matter Phys. 2, 141-165 (2011).

23. Takiguchi, K. et al. Quantum transport evidence of Weyl fermions in an epitaxial ferromagnetic oxide. Nat. Commun. 11, 4969 (2020).

24. Lin, W. et al. Electric field control of the magnetic Weyl fermion in an epitaxial $\mathrm{SrRuO}_{3}$ (111) thin film. Adv. Mater. 33, 2101316 (2021).

25. Zhang, Y., Ma, C., Lu, X. \& Liu, M. Recent progress on flexible inorganic singlecrystalline functional oxide films for advanced electronics. Mater. Horiz. 6, 911-930 (2019).

26. Zhang, B., Yun, C. \& MacManus-Driscoll, J. L. High yield transfer of clean large-area epitaxial oxide thin films. Nano-Micro Lett. 13, 1-14 (2013).

27. Lu, D. et al. Synthesis of freestanding single-crystal perovskite films and heterostructures by etching of sacrificial water-soluble layers. Nat. Mater. 15, 1255-1260 (2016).

28. Hong, S. S. et al. Two-dimensional limit of crystalline order in perovskite membrane films. Sci. Adv. 3, 5173 (2017).

29. Ji, D. et al. Freestanding crystalline oxide perovskites down to the monolayer limit. Nature 570, 87-90 (2019).

30. Dong, G. et al. Super-elastic ferroelectric single-crystal membrane with continuous electric dipole rotation. Science 366, 475-479 (2019).

31. Hong, S. S. et al. Extreme tensile strain states in $\mathrm{La}_{0.7} \mathrm{Ca}_{0.3} \mathrm{MnO}_{3}$ membranes. Science 368, 71-76 (2020).

32. Lu, D., Crossley, S., Xu, R., Hikita, Y. \& Hwang, H. Y. Freestanding oxide ferroelectric tunnel junction memories transferred onto silicon. Nano Lett. 19, 3999-4003 (2019).

33. $\mathrm{Xu}, \mathrm{R}$. et al. Strain-induced room-temperature ferroelectricity in $\mathrm{SrTiO}_{3}$ membranes. Nat. Commun. 11, 3141 (2020).

34. Pesquera, D. et al. Large magnetoelectric coupling in multiferroic oxide heterostructures assembled via epitaxial lift-off. Nat. Commun. 11, 3190 (2020).

35. Le, P. T. P., ten Elshof, J. E. \& Koster, G. Epitaxial lift-off of freestanding (011) and (111) $\mathrm{SrRuO}_{3}$ thin films using a water sacrificial layer. Sci. Rep. 11, 12435 (2021).

36. Han, K. et al. Enhanced metal-insulator transition in freestanding $\mathrm{VO}_{2}$ down to 5 $\mathrm{nm}$ thickness. ACS Appl. Mater. Interfaces 13, 16688-16693 (2021).

37. Lu, Z. et al. Synthesis of single-crystal $\mathrm{La}_{0.67} \mathrm{Sr}_{0.33} \mathrm{MnO}_{3}$ freestanding films with different crystal-orientation. APL Mater. 8, 051105 (2020).

38. Shrestha, S. et al. Nanometer-thick $\mathrm{Sr}_{2} \mid \mathrm{IO}_{4}$ freestanding films for flexible electronics. ACS Appl. Nano Mater. 3, 6310-6315 (2020).
39. $\mathrm{Hu}, \mathrm{C}$-Z. et al. Strain-controlled electrical and magnetic properties of $\mathrm{SrRuO}_{3}$ thin films with $\mathrm{Sr}_{3} \mathrm{Al}_{2} \mathrm{O}_{6}$ buffer layers. Appl. Phys. Lett. 118, 072407 (2021).

40. Lu, D. et al. Strain tuning in complex oxide epitaxial films using an ultrathin strontium aluminate buffer layer. Phys. Status Solidi RRL 12, 1700339 (2018).

41. Zhou, J. et al. Modulation of spin-orbit torque from $\mathrm{SrRuO}_{3}$ by epitaxial-straininduced octahedral rotation. Adv. Mater. 33, 2007114 (2021).

42. Gao, R. et al. Interfacial octahedral rotation mismatch control of the symmetry and properties of $\mathrm{SrRuO}_{3}$. ACS Appl. Mater. Interfaces 8, 14871-14878 (2016).

43. Lu, W., Yang, P., Song, W. D., Chow, G. M. \& Chen, J. S. Control of oxygen octahedral rotations and physical properties in $\mathrm{SrRuO}_{3}$ films. Phys. Rev. B 88, 214115 (2013).

44. Zeng, Z. et al. Emergent ferromagnetism with tunable perpendicular magnetic anisotropy in short-periodic $\mathrm{SrlrO}_{3} / \mathrm{SrRuO}_{3}$ superlattices. Appl. Phys. Lett. 116 142401 (2020).

45. Wei, J. et al. Enhancement of spin-orbit torque by strain engineering in $\mathrm{SrRuO}_{3}$ films. Adv. Funct. Mater. 31, 2100380 (2021).

46. Jeong, S. G. et al. Propagation control of octahedral tilt in $\mathrm{SrRuO}_{3}$ via artificial heterostructuring. Adv. Sci. 7, 2001643 (2020).

47. Herklotz, A. \& Dörr, K. Characterization of tetragonal phases of $\mathrm{SrRuO}_{3}$ under epitaxial strain by density functional theory. Eur. Phys. J. B 88, 1-5 (2015).

48. Ishigami, $K$. et al. Thickness-dependent magnetic properties and strain-induced orbital magnetic moment inSrRuO 3 thin films. Phys. Rev. B 92, 064402 (2015).

49. $\mathrm{Yi}, \mathrm{D}$. et al. Atomic-scale control of magnetic anisotropy via novel spin-orbit coupling effect in $\mathrm{La}_{2 / 3} \mathrm{Sr}_{1 / 3} \mathrm{MnO}_{3} / \mathrm{SrlrO}_{3}$ superlattices. Proc. Natl Acad. Sci. 113, 6397-6402 (2016).

50. Shen, L. et al. Epitaxial lift-off of centimeter-scaled spinel ferrite oxide thin films for flexible electronics. Adv. Mater. 29, 1702411 (2017).

51. Lang, L. et al. A low temperature functioning $\mathrm{CoFeB} / \mathrm{MgO}$-based perpendicular magnetic tunnel junction for cryogenic nonvolatile random access memory. Appl. Phys. Lett. 116, 022409 (2020).

52. Garzón, E. et al. Relaxing non-volatility for energy-efficient DMTJ based cryogenic STT-MRAM. Solid-State Electron. 184, 108090 (2021).

53. Kresse, G. \& Hafner, J. Ab initio molecular dynamics for liquid metals. Phys. Rev. $B$ 47, 558-561 (1993)

54. Kresse, G. \& Furthmiiller, J. Efficiency of ab-initio total energy calculations for metals and semiconductors using a plane-wave basis set. Comput. Mater. Sci. 6 , 15-50 (1996).

55. Kresse, G. \& Furthmiiller, J. Efficient iterative schemes for ab initio total-energy calculations using a plane-wave basis set. Phys. Rev. B 54, 11169 (1996).

56. Perdew, J. P., Burke, K. \& Ernzerhof, M. Generalized gradient approximation made simple. Phys. Rev. Lett. 77, 3865-3868 (1996).

57. Anisimov, V. I., Aryasetiawan, F. \& Lichtenstein, A. I. First-principles calculations of the electronic structure and spectra of strongly correlated. J. Phys.: Condens. Matter 9, 767-808 (1997).

\section{ACKNOWLEDGEMENTS}

This work was supported by the National Key Research and Development Program of China (Nos. 2017YFA0303600, 2019YFA0307800), the National Natural Science Foundation of China (Nos. 12174406, U1832102, 11874367, 51931011, 51902322), the Key Research Program of Frontier Sciences, Chinese Academy of Sciences (No. ZDBS-LY-SLH008), the Thousand Young Talents Program of China, K. C. Wong Education Foundation (GJTD-2020-11), the 3315 Program of Ningbo, the Natural Science Foundation of Zhejiang province of China (No. LR20A040001), the Public Welfare Technical Applied Research Project of Zhejiang Province (No. LY21E020007), the Ningbo Natural Science Foundation (No. 2019A610050), the Beijing National Laboratory for Condensed Matter Physics.

\section{AUTHOR CONTRIBUTIONS}

Z.W. designed the experiments. Y.Y. and B.C. synthesized the targets. Z.L., Y.Y., and Z.R. performed sample growth. Z.L., Y.Y., S.L., and K.N. performed magnetism measurements. Z.L., Y.Y., X.Z., D.S., and H.D. performed sample structural characterization and data analysis. L.W. and X.H. performed theoretical calculations. Z.L. wrote the manuscript with significant contributions from J.F., Y.G., Z.Z, X.H., Z.W. and R.-W.L. as well as contributions from all other authors. Z.W. and R.-W.L. supervised the project.

\section{COMPETING INTERESTS}

The authors declare no competing interests. 


\section{ADDITIONAL INFORMATION}

Supplementary information The online version contains supplementary material available at https://doi.org/10.1038/s41528-022-00141-3.

Correspondence and requests for materials should be addressed to Xianfeng Hao or Zhiming Wang.

Reprints and permission information is available at http://www.nature.com/ reprints

Publisher's note Springer Nature remains neutral with regard to jurisdictional claims in published maps and institutional affiliations.
Open Access This article is licensed under a Creative Commons Attribution 4.0 International License, which permits use, sharing, adaptation, distribution and reproduction in any medium or format, as long as you give appropriate credit to the original author(s) and the source, provide a link to the Creative Commons license, and indicate if changes were made. The images or other third party material in this article are included in the article's Creative Commons license, unless indicated otherwise in a credit line to the material. If material is not included in the article's Creative Commons license and your intended use is not permitted by statutory regulation or exceeds the permitted use, you will need to obtain permission directly from the copyright holder. To view a copy of this license, visit http://creativecommons. org/licenses/by/4.0/.

(c) The Author(s) 2022 